\title{
On the shape of energy-detection ROC curves
}

\author{
GLENN L. GREIG \\ University of Waterloo, Waterloo, Ontario, Canada
}

\begin{abstract}
A numerical study shows that energy-detection ROC curves based on chi-squared distributions may be accurately represented by binormal receiver operating characteristic $(\mathrm{ROC})$ curves. This allows the detector accuracy and the ROC shape (asymmetry) to be expressed simply in terms of the distribution parameters. An established approximate formula for the shape of energydetection ROC curves is shown to be inaccurate. An improved approximation is presented.
\end{abstract}

Signal detection theory is a very useful tool for describing and predicting human performance in detection tasks. In the signal detection scenario, a stimulus is modeled as a signal in a background of random noise. The observer's task is to determine the presence or absence of a signal, on the basis of a noisy observation. The main results of a signal detection experiment or analysis can be represented graphically by the receiver operating characteristic, or ROC, curve.

For any detection model, it would be useful to have a concise description of the ROC curve that is meaningful in terms of the underlying signal and noise distributions. Such a description would facilitate the comparison of model with experiment, and help in the assessment of the effect of changing model parameters. In this paper, I shall present such a description for ROC curves that are based on chi-squared distributions of signal and noise. This case arises most commonly from an energy-detection model.

\section{Review and Notation}

The mathematics of signal detection theory are described in detail by Green and Swets (1974). Briefly, signal detection theory models the observer as a two-stage signal processor. The first stage processes the input stimulus to produce a scalar evidence statistic. This statistic, $e$, is a random variable whose distribution is conditional upon the presence or absence ( $s$ or $n$ ) of a signal. The second stage makes a statistical decision by comparing $e$ to some decision criterion $k$. In the simplest case, the decision rule is to respond $S$ (a signal was present) if $e>k$, or $N$ (a signal was not present) if $e<k$.

The ROC curve is obtained by plotting the probability of a correct detection, $P(S \mid s)$, versus the probability of a false alarm, $P(S \mid n)$, for all values of $k$. The ROC curve is related to the conditional distributions of $e, f(e \mid n)$ and $f(e \mid s)$, by the following equations. For any value of $k$,

This work was carried out while the author was at the University of Toronto Institute for Aerospace Studies and was supported by an NSERC postgraduate fellowship. Correspondence should be addressed to Glenn L. Greig, Department of Systems Design Engineering, University of Waterloo, Waterloo, Ontario N2L 3G1, Canada.

$$
\begin{aligned}
& P(S \mid n)=\int_{k}^{\infty} f(e \mid n) d e \\
& P(S \mid s)=\int_{k}^{\infty} f(e \mid s) d e
\end{aligned}
$$

Different detection models lead to different assumptions about the form of $f(e \mid n)$ and $f(e \mid s)$ for a given physical description of signal and noise. Therefore, experimentally determined ROC curves can provide evidence for or against particular detection models. This comparison of model with experiment is facilitated if we have a concise description of the form of ROC curves predicted by our detection model.

\section{Representation of Binormal ROC Curves}

A simple and precise description of the ROC curve is available for the case in which $f(e \mid n)$ and $f(e \mid s)$ are Gaussian. Swets and Pickett (1982) refer to such ROC curves as binormal ROC curves. A binormal ROC curve is a straight line when plotted on normal probability axes (that is, on a binormal graph). Therefore, it can be described exactly by two parameters: a slope and an intercept.

It can easily be shown (Green \& Swets, 1974) that the slope of the line is given by:

$$
b=\sigma_{n} / \sigma_{s},
$$

where $\sigma_{n}$ and $\sigma_{s}$ are the standard deviations of $f(e \mid n)$ and $f(e \mid s)$, respectively. This shape parameter, $b$, is an indicator of the asymmetry of the ROC curve. If $b=1$, the ROC curve (plotted on linear axes) is symmetric about the line from $(0,1)$ to $(1,0)$. If $b \neq 1$, then the ROC curve is asymmetrical. Figure 1 shows two typical ROC curves with $b<1$.

The second parameter provides a measure of the overall accuracy of the detector. For the case where $\sigma_{n}=\sigma_{s}$, the usual choice for this parameter is the detectability index:

$$
d^{\prime}=\left(\mu_{s}-\mu_{n}\right) / \sigma_{n},
$$

where $\mu_{s}$ and $\mu_{n}$ are the means of $f(e \mid s)$ and $f(e \mid n)$, respectively.

Several accuracy parameters have been defined for the case in which $\sigma_{n} \neq \sigma_{s}$ (Swets \& Pickett, 1982). The 


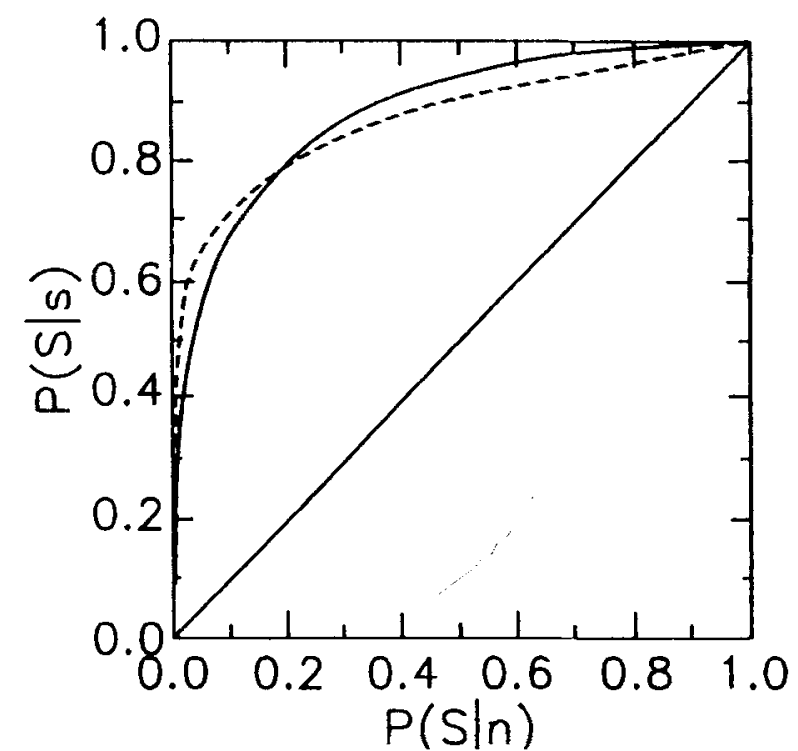

Figure 1. Binormal ROC curves with $b=0.87$ (solid line), and $b=0.58$ (dotted line).

measure preferred by this author is a modified detectability index:

$$
d_{A}=\left(\mu_{s}-\mu_{n}\right) / \sqrt{\frac{1}{2}\left(\sigma_{n}^{2}+\sigma_{s}^{2}\right)} .
$$

The merit of this measure is that it is consistent with another (nonparametric) accuracy measure: the area under the ROC curve, $P_{A} . d_{A}$ is the same for all binormal ROC curves with the same value of $P_{A}$ (it is independent of b). Note that $d_{A}=d^{\prime}$ when $\sigma_{n}=\sigma_{s}$.

This two-parameter description is so useful that we are tempted to use it even when $f(e \mid n)$ and $f(e \mid s)$ are not actually Gaussian. If an ROC curve is approximately linear when plotted on a binormal graph, we can describe it by the two parameters that define the best-fitting binormal ROC curve. It is shown below that energy-detection ROC curves are approximately binormal. Approximate expressions for $d_{A}$ and $b$ are presented.

\section{Energy-Detection ROC Curves}

For a broad class of detection problems, the optimal detection rule is based on the total energy of the input stimulus. For example, Green and Swets (1974, chap. 8) showed that an energy detector was optimal for the detection of poorly defined temporal signals in the presence of band-limited Gaussian noise. An energy-detection model often leads to chi-squared distributions of signal and noise.

For the case noted above, Green and Swets (1974) defined $e$ to be the total input energy over the observation interval, and showed that $f(e \mid n)$ is chi-squared with $N$ degrees of freedom $(d f)$, and $f(e \mid s)$ is noncentral chisquared with $N d f$ and noncentrality parameter $\delta . N$ and $\delta$ depend on the physical characteristics of signal and noise: $N=2 W T$, where $W$ is the noise bandwidth and
$T$ is the duration of the observation interval; and $\delta=2 E_{s} / N_{o}$ is the signal-to-noise ratio.

The probability of a false alarm and a correct detection may now be expressed in terms of the chi-squared distributions. For any value of the criterion, $k$ :

$$
\begin{aligned}
& P(S \mid n)=\int_{k}^{\infty} f(e \mid n) d e=1-P_{x^{2}}(k \mid N) \\
& P(S \mid s)=\int_{k}^{\infty} f(e \mid s) d e=1-P_{x^{2^{\prime}}}(k \mid N, \delta)
\end{aligned}
$$

where $P_{\chi^{2}}(k \mid N)$ is the cumulative chi-squared distribution with $N d f$, and $P_{x^{2}}(k \mid N, \delta)$ is the cumulative noncentral chi-squared distribution with $N d f$ and noncentrality parameter $\delta$. These are defined by Abramowitz and Stegun (1964, Equations 26.4.1 and 26.4.25) as:

$$
\begin{aligned}
P_{x^{2}}(k \mid N) & =\left[2^{N / 2} \Gamma(N / 2)\right]^{-1} \int_{0}^{k} t^{N / 2-1} e^{-t / 2} d t \\
P_{\chi^{2^{\prime}}}(k \mid N, \delta) & =\sum_{j=0}^{\infty} \exp (-\delta / 2) \frac{(\delta / 2)^{j}}{j !} P_{\chi^{2}}(k \mid N+2 j)
\end{aligned}
$$

The form of these distributions is best shown graphically. The signal and noise distributions for a typical case, with $N=10$ and $\delta=10$, are shown in Figure 2. The corresponding ROC curve, plotted on linear axes, is the solid curve shown in Figure 1.

\section{Binormal Approximation to \\ Energy-Detection ROC Curves}

We can use Equations 6 to 9 to obtain the ROC curve for any given condition. However, it is not easy to see how the detectability and ROC shape depend on the distribution parameters, $N$ and $\delta$. A simple description of the ROC curve in terms of $N$ and $\delta$ would be very useful.

Green and Swets (1974) address this problem. They argue that when $N>10$, the chi-squared distributions are

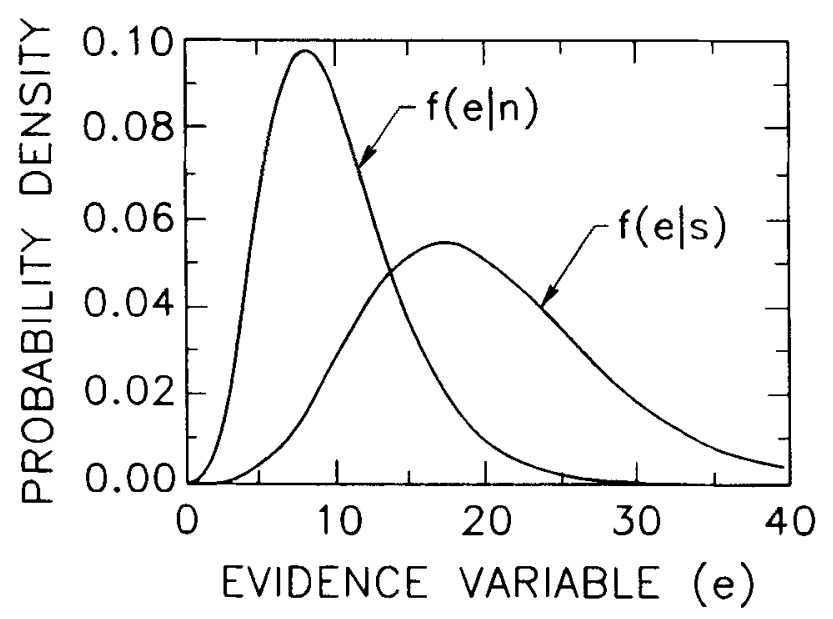

Figure 2. Chi-squared signal and noise distributions for the case where $N=10$ and $\delta=10$. 
approximately normal, and they suggest that the resulting ROC curve may be approximated by a binormal ROC curve. They are not very clear on how to proceed, but their analysis (pp. 219 and 224) implies that the chisquared distributions should simply be replaced by normal distributions with the same mean and variance. The mean and variance of the chi-squared distributions are:

$$
\begin{array}{ll}
\mu_{n}=N & \mu_{s}=N+\delta \\
\sigma_{n}^{2}=2 N & \sigma_{s}^{2}=2 N+4 \delta
\end{array}
$$

Using these values in Equations 5 and 3 yields the following approximations for $d_{A}$ and $b$ :

$$
\begin{gathered}
d_{A} \doteq d_{1}=\delta / \sqrt{2(N+\delta)} \\
b \doteq b_{1}=\sigma_{n} / \sigma_{s}=\sqrt{2 N /(2 N+4 \delta)}
\end{gathered}
$$

These relations are approximate, since they are based on Gaussian approximations to chi-squared distributions. However, they are useful in showing how the detectability and ROC shape depend on $N$ and $\delta$. For example, Equation 12 implies that the asymmetry of the ROC curve will increase with a decrease in $N$. Green and Swets (1974) discuss this in the context of critical bands for auditory detection: Since $N=2 W T$, a narrower critical band $(W)$ implies a more asymmetrical ROC curve.

\section{A New Approximation for the Shape Parameter}

It became apparent in a recent study by Greig (1988) that energy-detection ROC curves may not be as asym- metrical as is indicated by Equation 12. A numerical study was carried out to check the validity of Equation 12 .

The numerical study covered a range of the two model parameters: $N$, the number of degrees of freedom, and $\delta$, the noncentrality parameter. For each pair of parameters $(N, \delta), 8$ points on the ROC curve were generated by applying Equations 6 to 9 for eight values of $k$. The points were chosen to be more or less evenly spaced, covering the range from $P(S \mid s)=0.1$ to $P(S \mid n)=0.9$. For each point, Equation 8 and the embedded chi-squared distribution in Equation 9 were evaluated with an accurate algorithm from Press, Flannery, Teukolsky, \& Vetterling, 1988 , p. 177).

A maximum-likelihood estimation algorithm based on Dorfman and Alf (1969) was used to fit an ROC curve to each set of points. The algorithm assumes that $f(e \mid n)$ and $f(e \mid s)$ are Gaussian, and calculates the slope and intercept of the line that best fits the set of points, plotted on a binormal graph. These maximum-likelihood or bestfit parameters were then compared with the estimates of $d_{A}$ and $b$ found using Equations 11 and 12.

The results for a typical case are shown in Figures 2 and 3. The signal and noise distributions with $N=10$ and $\delta=10$ are shown in Figure 2. Figure 3 is a binormal graph of the ROC curve. The symbols in Figure 3 are points on the true ROC curve. The solid line is the binormal ROC curve based on the maximum-likelihood estimates of $d_{A}$ and $b$. The dotted line is the binormal ROC curve based on $d_{1}$ and $b_{1}$ (Equations 11 and 12). These two curves are also shown on a linear ROC graph in Figure 1.

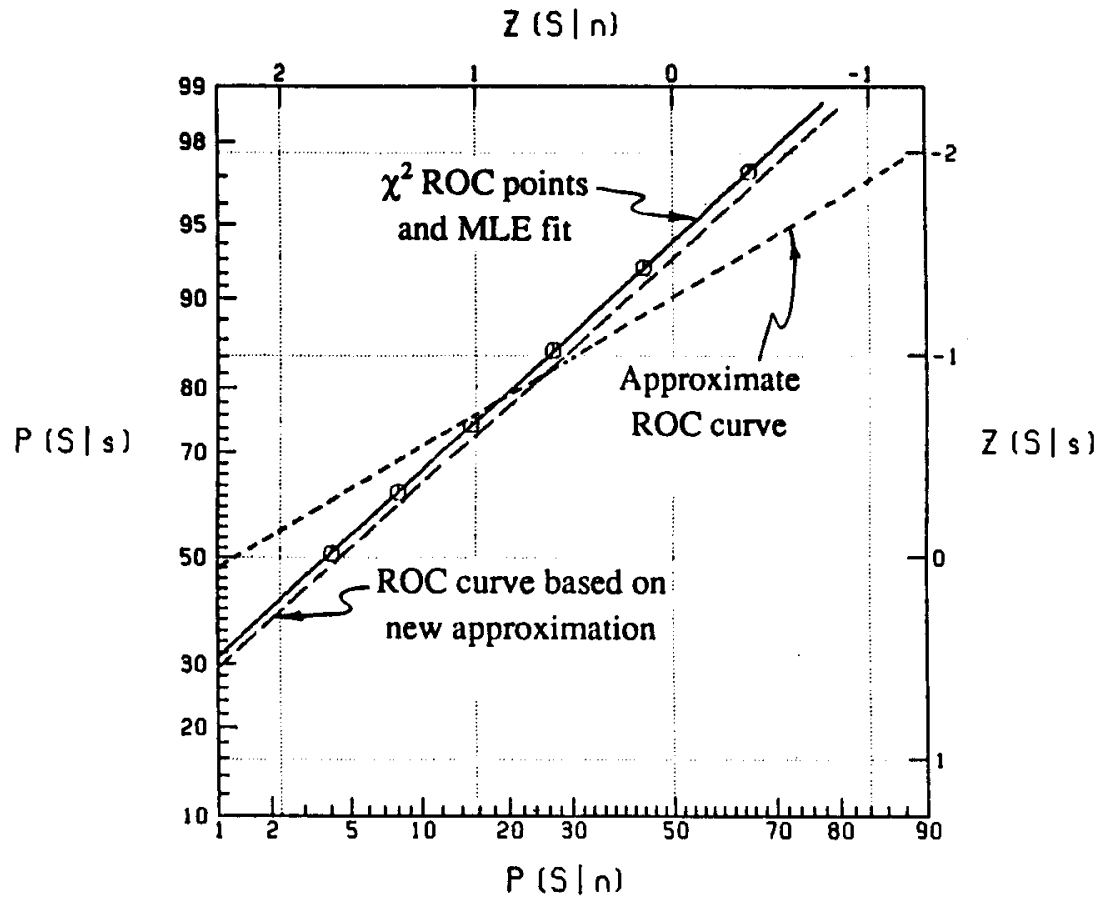

Figure 3. Binormal approximations to the ROC curve based on chi-squared distributions with $N=10$ and $\delta=10$. 
The maximum-likelihood ROC curve fits the points very closely, indicating that this energy-detection ROC curve (based on chi-squared distributions) is almost exactly linear on a binormal graph. However, the ROC curve based on Equations 11 and 12 does not fit the points well. This indicates that these approximations are inaccurate. In particular, Equation 12 significantly underestimates the shape parameter, $b$. Similar results were obtained for the entire range of parameters studied. These results are summarized in Table 1.

It can be seen from Table 1 that the error in $b_{1}$ increases with $(1-b)$. This suggests that a better estimate of $b$ might be obtained using a power law of the form:

$$
b_{2}=b_{1}^{\alpha}, \quad \alpha<1
$$

or a linear relationship of the form:

$$
b_{3}=1-c\left(1-b_{1}\right), \quad c<1 \text {. }
$$

The best agreement between these approximations and the maximum-likelihood estimate of $b$ was obtained by setting $\alpha=0.25$ in Equation 13 , and $c=0.30$ in Equation 14. The results are shown in Table 1 . Both $b_{2}$ and $b_{3}$ are good estimators of $b$; the error is generally within $1 \%$. This agreement was achieved over a representative range of parameter pairs $(N, \delta)$, and it should hold for any values found in practice.

Equation 11 provides a reasonably accurate estimate of $d_{A}$, except for low values of $\sigma_{n} / \sigma_{s}$. When $\sigma_{n} / \sigma_{s}<0.6$, $d_{1}$ may underestimate the true detectability by $5 \%-10 \%$. However, Equation 11 is accurate within 3\% for most of the conditions tested. This error is small compared with typical errors in experimental estimates of detectability. Therefore, an energy-detection ROC curve may be adequately described by a binormal ROC curve, with $d_{A}$ given

Table 1

Detectability and Binormal Slope Estimates

\begin{tabular}{|c|c|c|c|c|c|c|c|}
\hline \multirow[b]{2}{*}{$N$} & \multirow[b]{2}{*}{$\delta$} & \multicolumn{2}{|c|}{ Detectability, $d_{A}$} & \multicolumn{4}{|c|}{ Binormal ROC Slope, $b$} \\
\hline & & $M L E$ & $d_{1}$ & $M L E$ & $b_{1}$ & $b_{2}$ & $b_{3}$ \\
\hline 3 & $\begin{array}{l}1.5 \\
3.0 \\
5.0\end{array}$ & $\begin{array}{r}.496 \\
.900 \\
1.357\end{array}$ & $\begin{array}{r}.500 \\
.866 \\
1.250\end{array}$ & $\begin{array}{l}.907 \\
.870 \\
.846\end{array}$ & $\begin{array}{l}.707 \\
.577 \\
.480\end{array}$ & $\begin{array}{l}.917 \\
.872 \\
.833\end{array}$ & $\begin{array}{l}.912 \\
.873 \\
.844\end{array}$ \\
\hline 5 & $\begin{array}{l}2.0 \\
4.0 \\
7.0\end{array}$ & $\begin{array}{r}.533 \\
.972 \\
1.532\end{array}$ & $\begin{array}{r}.535 \\
.943 \\
1.429\end{array}$ & $\begin{array}{l}.921 \\
.888 \\
.864\end{array}$ & $\begin{array}{l}.745 \\
.620 \\
.513\end{array}$ & $\begin{array}{l}.929 \\
.887 \\
.846\end{array}$ & $\begin{array}{l}.924 \\
.886 \\
.854\end{array}$ \\
\hline 10 & $\begin{array}{r}3.0 \\
6.0 \\
10.0\end{array}$ & $\begin{array}{r}.588 \\
1.084 \\
1.658\end{array}$ & $\begin{array}{r}.588 \\
1.061 \\
1.581\end{array}$ & $\begin{array}{l}.937 \\
.906 \\
.884\end{array}$ & $\begin{array}{l}.791 \\
.674 \\
.577\end{array}$ & $\begin{array}{l}.943 \\
.906 \\
.872\end{array}$ & $\begin{array}{l}.937 \\
.902 \\
.873\end{array}$ \\
\hline 30 & $\begin{array}{r}5.0 \\
10.0 \\
16.0\end{array}$ & $\begin{array}{r}.597 \\
1.128 \\
1.703\end{array}$ & $\begin{array}{r}.598 \\
1.118 \\
1.668\end{array}$ & $\begin{array}{l}.959 \\
.934 \\
.914\end{array}$ & $\begin{array}{l}.866 \\
.775 \\
.696\end{array}$ & $\begin{array}{l}.965 \\
.938 \\
.913\end{array}$ & $\begin{array}{l}.960 \\
.932 \\
.909\end{array}$ \\
\hline 60 & $\begin{array}{r}6.0 \\
12.0 \\
20.0\end{array}$ & $\begin{array}{r}.522 \\
1.003 \\
1.597\end{array}$ & $\begin{array}{r}.522 \\
1.000 \\
1.581\end{array}$ & $\begin{array}{l}.972 \\
.953 \\
.934\end{array}$ & $\begin{array}{l}.913 \\
.845 \\
.775\end{array}$ & $\begin{array}{l}.977 \\
.959 \\
.938\end{array}$ & $\begin{array}{l}.974 \\
.954 \\
.932\end{array}$ \\
\hline 120 & 16.0 & .972 & .970 & .965 & .889 & .971 & .967 \\
\hline
\end{tabular}
for ROC Curves Based on Chi-squared Distributions

Note-MLE is the maximum-likelihood estimate. $d_{1}, b_{1}, b_{2}$, and $b_{3}$ are approximate values given by Equations $11,12,13$, and 14 , respectively.

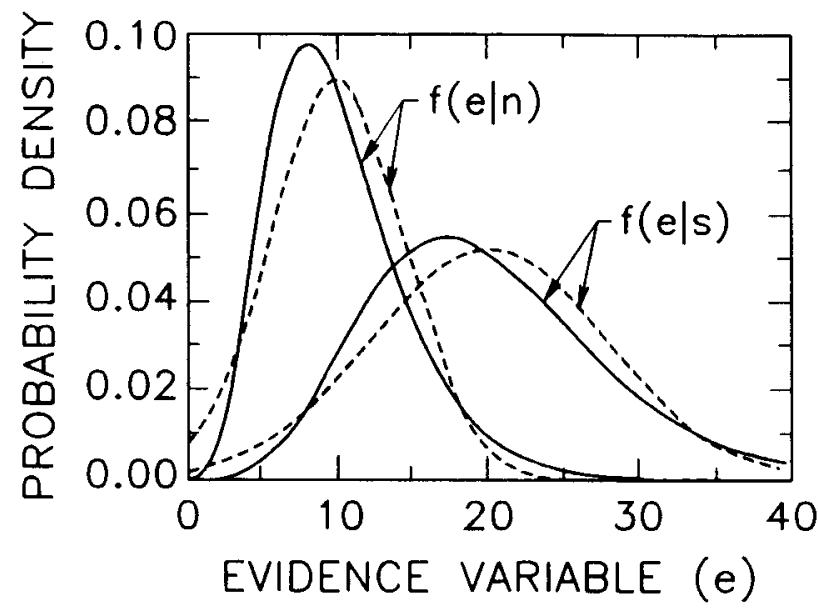

Figure 4. Chi-squared signal and noise distributions for the case where $N=10$ and $\delta=10$, and normal distributions with the same means and variances.

by Equation 11 , and $b$ given by Equation 13 or Equation 14. The dashed line in Figure 3 shows the binormal ROC curve based on Equations 11 and 13 for the example case.

\section{Qualitative Explanation of the Results}

Equations 11 and 12 are based on the assumption that chi-squared distributions may be represented by Gaussian distributions with the same mean and variance. Why does this assumption lead to an inaccurate estimate of $b$ ? The error appears to be due to the skewness of the chi-squared distributions.

Figure 4 shows a comparison of the chi-squared distributions of Figure 2 with the normal distributions having the same means and variances. Both chi-squared distributions have positive skewness, which means, essentially, that the right-hand or positive tail of the distribution is longer than the left-hand tail. The normal distributions, of course, are symmetrical.

At high values of $e$ [low values of $P(S \mid n)$ ], the normal approximation to $f(e \mid n)$ drops off too fast with increasing $e$. This results in an underestimation of $P(S \mid n)$ for a given value of $P(S \mid s)$; the estimated ROC point is above the true ROC curve. Conversely, at low values of $e$ [high values of $P(S \mid s)$ ], the normal approximation to $f(e \mid s)$ drops off too slowly with decreasing $e$. In this range, the approximation produces estimated ROC points that are below the true ROC curve. The net effect is that the normal approximation leads to an underestimation of the binormal slope, as is shown in Figure 3.

This argument implies that the error in Equation 12 should increase with the skewness of the distributions. Abramowitz and Stegun (1964, p. 943) show that the skewness coefficient of chi-squared distributions decreases with increasing $N$. Therefore, the error in Equation 12 should be greatest at low values of $N$. The data in Table 1 confirm this. 


\section{Conclusion}

Any energy-detection ROC curve (described by chisquared distributions) may be closely fit by an equivalent binormal ROC curve. The detectability index for the detector may be found using Equation 11. However, the ratio $\sigma_{n} / \sigma_{s}$ (Equation 12) does not accurately estimate the slope of the equivalent binormal ROC curve. This estimate is inaccurate, because it does not account for the skewness of the chi-squared distributions; energy-detection ROC curves are much less asymmetrical than is suggested by Equation 12. This result should also to apply to ROC curves based on other skewed distributions such as the Poisson and gamma.

Two simple empirical approximations for the slope of the equivalent binormal ROC curve are presented (Equations 13 and 14); both are accurate within about $1 \%$ over a wide range. These approximations will be of value in comparing experimental ROC curves with model predictions, or generating ROC curves based on limited data.

\section{REFERENCES}

ABramowitz, M., \& STEgUN, I. A. (1964). Handbook of mathematical functions (Applied Mathematics Series, Vol, 55). Washington, DC: National Bureau of Standards.

Dorfman, D. D., \& ALF, E., JR. (1969). Maximum likelihood estimation of parameters of signal-detection theory and determination of confidence intervals-rating method data. Joumal of Mathematical Psychology, 6, 487-496.

Green, D. M., \& Swets, J. A. (1974). Signal detection theory and psychophysics. New York: Wiley.

GREIG, G. L. (1988). Masking of motion cues by random motion: Comparison of human performance with a signal detection model (UTLAS Report No. 313). Toronto: University of Toronto Institute for Aerospace Studies.

Press, W. H., Flannery, B. P., Teukolsky, S. A., VetterLING, W. T. (1988). Numerical recipes in C. Cambridge, England: Cambridge University Press.

SWETs, J. A., PICKeTt, R. M. (1982). Evaluation of diagnostic systems: Methods from signal detection theory. New York: Academic Press.

(Manuscript received August 28, 1989; revision accepted for publication January 30,1990 .) 